\title{
Quantifying the regeneration of bone tissue in biomedical images via Legendre moments
}

\author{
José A. Lachiondo \& Manuel Ujaldón \\ Computer Architecture Department \\ University of Malaga, Spain
}

Regina Berretta \& Pablo Moscato

Centre for Bioinformatics, Biomarker Discovery and Information-based Medicine

University of Newcastle, Australia

\begin{abstract}
We investigate the use of Legendre moments as biomarkers for an efficient and accurate classification of bone tissue on images coming from stem cell regeneration studies. Regions of either existing bone, cartilage or new bone-forming cells are characterized at tile level to quantify the degree of bone regeneration depending on culture conditions. Legendre moments are analyzed from three different perspectives: (1) their discriminant properties in a wide set of preselected vectors of features based on our clinical and computational experience, providing solutions whose accuracy exceeds 90\%. (2) the amount of information to be retained when using Principal Component Analysis (PCA) to reduce the dimensionality of the problem from 2 to 6 dimensions. (3) the use of the $(\alpha-\beta)$ - $k$-feature set problem to identify a $k=4$ number of features which are more relevant to our analysis from a combinatorial optimization approach. These techniques are compared in terms of computational complexity and classification accuracy to assess the strengths and limitations of the use of Legendre moments for this biomedical image processing application.
\end{abstract}

\section{INTRODUCTION}

Spatial domain filters are the most direct way to characterize edges, granularity and shape as global features within image analysis. An important subset of spatial domain filters are based on moment functions of pixel intensity values (9). These functions are expressed as discrete sums, with some of them being invariant under image translation, scale change and rotation $(2,4)$. Moments with orthogonal basis functions such as Legendre and Zernike polynomials are even more useful as image descriptors because they can represent the image by a set of mutually independent descriptors, with a minimal amount of information redundancy (8).

In this work, we analyze the power of Legendre moments as new biomarkers for tracking the evolution of bone-forming cells from pluripotent mesenchymal stem cells (1). To make our application compatible with other cases like X-ray or electronic microscope images which work with gray-scales, our techniques are applied over the luminance, which merges the three colors into a single channel.

Each of our input images is a slice 7 microns thick containing 2,560x1,920 pixels, which is decomposed into 40x30 tiles of $64 \times 64$ pixels for its further characterization into three different tissue types: Existing bone, cartilage and regenerated bone. The ultimate goal is to quantify the degree of bone regeneration in tissue depending on culture conditions, and identify those that best contribute to the induction of boneforming cells from stem cells.

For each environmental conditions to be analyzed, input data is around 1.5 Gpixels and each pixel requires intensive processing, leading to a heavy workload. This fact motivated us to carry out a threefold effort to accelerate the process:

1. A pre-selection of Legendre moments is described in Section 3 based on computational complexity and previous experiences (5).

2. The dimensionality of the problem is shortened using Principal Component Analysis (PCA) in Section 4 prior to the actual classification process, whose success is evaluated in Section 6.

3. The $(\alpha-\beta)$ - $k$-feature set (3) is a combinatorial optimization problem we describe in Section 5 to identify the more discriminant moments. 


\section{LEGENDRE MOMENTS}

The theory of moments (8) provides a convenient way of characterizing patterns within images. We are interested in using them as descriptors for each of the tiles of $64 \times 64$ pixels in which our biomedical images are decomposed. Zernike moments were already analyzed within this context (5), also to justify the tile size. In this work, we focus on Legendre moments.

Legendre polynomials are orthogonal basis functions which allow to represent an image by a set of independent features with minimal information redundancy. The image can thus be reconstructed from the linear addition of moments (and integral ideally), and when computational requirements need to be relaxed, the image can be roughly characterized by a limited set of those moments.

To derive a computational formula, we must discretize the general approach by switching integrals into discrete sums and approximate Legendre moments of order $p$ and repetition $q, A_{p q}$, as follows:

$A_{p q}=\frac{(2 q+1)(2 p+1)}{4} \sum_{x=1}^{64} \sum_{y=1}^{64} P_{q}(x) P_{p}(y) P_{x y}$

where $x$ and $y$ are the pixel coordinates, $P_{x y}$ is the pixel intensity value (between 0 and 255 for the case of our image format), and $P_{i}(x)$ is the Legendre polynomial of $\mathrm{i}^{\text {th }}$ order, typically defined as:

$P_{i}(x)=\sum_{j=0}^{i} a_{i j} x^{j}$

and $a_{i j}$ are the Legendre coefficients given by:

$a_{i j}=(-1)^{\frac{(i-j)}{2}} \frac{1}{2^{i}} \frac{(i+j) !}{\frac{(i-j)}{2} !\left(\frac{(i+j)}{2} ! j !\right.}, \quad p-q=\operatorname{even}(3)$

Legendre moments are not invariant under linear transformations or rotation, which makes them less suitable than Zernike moments for certain image analysis applications, but when these drawbacks are overcome, they relax the computational cost (7).

Our next step would be to calculate all orthogonal Legendre moments up to an order $o$ and repetition $r$ within an image tile, and represent such tile by its vector of $\mathrm{F}=o \cdot r$ features (the image space), to use the F-dimensional vector as tile descriptor for the subsequent classification process. Nevertheless, we will reduce the computational complexity in three basic ways: With a pre-selection of moments in Section 3 , reducing the dimensionality of the problem using Principal Component Analysis (PCA) in Section 4, and using combinatorial optimization techniques to select the most discriminant moments in Section 5. Finally, Section 6 compares these methods in terms of computational complexity and accuracy.

\section{A PRELIMINARY SELECTION OF} MOMENTS FOR THE CLASSIFIER

The orthogonality property of Legendre moments enables the separation of the individual contribution of each order moment, so after a preliminary inspection, the candidate moments were limited to orders lower than 16 , since higher moments were found to be increasingly costly and irrelevant (they contribute to image reconstruction, which is far from being our goal here).

Based on the observation that the $A_{0,0}$ moment captures most of the variance between images of the classes, and helped by our previous experiences with Zernike moments (5), we pre-selected a set of 10 potential candidates as vectors of features used as input to our classifiers. They are illustrated in Table 2 with a correspondence matrix where the set of $A_{p q}$ moments composing each feature set used in a trial is marked with the $\bullet$ symbol, arranging candidate moments on rows and vectors of features on columns. Note that this table also contains some classification results in the right and lower margins which are worth of our attention on a later stage of this paper.

The rest of the classification process following this method involves the training samples as tiles to calculate the representative vectors for each class (centroids). These vectors are compared against those representing each of the input tiles, the vector norm is measured as distance, and tiles are assigned to the closest class.

\section{ANOTHER CLASSIFICATION PROCESS BASED ON PCA}

We use Principal Component Analysis (6) to reduce the dimensionality of the problem to help when realtime constraints and/or large-scale images are imposed by clinical practice. For a set of $T=30$ training tiles for each class in the data set and $F=17 x 17$ pre-selected Legendre moments which identify them, we create a $T x F$ matrix to reduce the dimensionality to $k=2,3,4,5$ or 6 dimensions in the final features space. This is attained through the following procedure:

\section{Calculate the FxF covariance matrix, D.}

2. Derive the F eigenvectors for D.

3. Diagonalize D to obtain its F eigenvalues.

4. Select the $k$ eigenvectors corresponding to the $k$ highest eigenvalues.

This produces a 2D subspace capturing most of the variations contained in the original image space, using the training samples as a representative data set. Now we take the whole input data set, and for each image tile identified by $F$ Legendre moments, we project it 
Table 1: The $A_{p q}$ Legendre moments (listed by rows) we used for classification purposes (vectors of features listed by columns). The penultimate column shows the percentage of classification success obtained when a particular moment is used as a single feature (alone) for the classifier (note that no PCA is required for those cases). The penultimate row shows the same percentage for each vector of features characterized. Rankings in last row/column indicate the position occupied by that vector/moment on a ranking built when sorting all candidate vectors/moments from highest to lowest successful percentage.

\begin{tabular}{|c|c|c|c|c|c|c|c|c|c|c|c|c|c|}
\hline \multicolumn{2}{|c|}{ Moment $A_{p, q}$} & \multicolumn{10}{|c|}{ Selection of moments for vectors of features } & \multirow{2}{*}{$\begin{array}{r}\text { Success } \\
\text { (alone) }\end{array}$} & \multirow{2}{*}{$\begin{array}{l}\text { Position in } \\
\text { our ranking }\end{array}$} \\
\hline $\mathrm{p}$ & $\mathrm{q}$ & 1 & 2 & 3 & 4 & 5 & 6 & 7 & 8 & 9 & 10 & & \\
\hline 0 & 0 & $\bullet$ & $\bullet$ & - & - & • & - & • & $\bullet$ & $\bullet$ & • & 92.0 & \#1 \\
\hline 1 & 1 & - & - & - & • & - & $\bullet$ & • & - & $\bullet$ & - & 22.6 & \#33 \\
\hline 2 & 2 & - & - & - & • & • & - & - & - & • & - & 49.0 & \#16 \\
\hline 3 & 3 & - & - & - & • & - & • & - & - & $\bullet$ & - & 44.0 & \#22 \\
\hline 4 & 4 & - & - & - & • & • & - & - & • & - & - & 46.3 & \#19 \\
\hline 5 & 5 & - & - & - & • & - & $\bullet$ & - & - & - & - & 38.0 & \#26 \\
\hline 6 & 6 & - & - & - & • & • & - & - & - & - & - & 38.6 & $\# 25$ \\
\hline 7 & 7 & - & - & - & $\bullet$ & - & $\bullet$ & - & - & - & - & 46.3 & \#19 \\
\hline 8 & 8 & - & - & - & • & • & - & - & $\bullet$ & - & - & 28.0 & \#32 \\
\hline 9 & 9 & - & - & - & - & - & • & - & - & - & - & 40.6 & \#23 \\
\hline 10 & 10 & - & - & - & • & • & - & - & - & - & - & 39.6 & $\# 24$ \\
\hline 11 & 11 & - & - & - & $\bullet$ & - & $\bullet$ & - & - & - & - & 50.6 & $\# 14$ \\
\hline 12 & 12 & - & - & - & • & • & - & - & • & - & - & 36.0 & \#28 \\
\hline 13 & 13 & - & - & - & - & - & • & - & - & - & - & 50.3 & $\# 15$ \\
\hline 14 & 14 & - & - & - & • & • & - & - & - & - & - & 35.6 & \#30 \\
\hline 15 & 15 & - & - & - & • & - & • & - & - & - & - & 54.3 & \#12 \\
\hline 16 & 16 & - & - & - & • & • & - & - & • & - & - & 49.0 & $\# 16$ \\
\hline 1 & 0 & $\bullet$ & - & $\bullet$ & - & - & - & • & - & - & - & 36.0 & \#28 \\
\hline 2 & 0 & • & • & - & - & - & - & - & • & - & - & 46.3 & \#19 \\
\hline 3 & 0 & $\bullet$ & - & • & - & - & - & - & - & - & - & 53.3 & $\# 13$ \\
\hline 4 & 0 & • & • & - & - & - & - & - & - & - & - & 37.6 & \#27 \\
\hline 5 & 0 & • & - & • & - & - & - & - & - & - & - & 65.3 & \#9 \\
\hline 6 & 0 & • & • & - & - & - & - & - & • & - & - & 30.0 & \#31 \\
\hline 7 & 0 & $\bullet$ & - & $\bullet$ & - & - & - & - & - & - & - & 74.6 & \#7 \\
\hline 8 & 0 & • & $\bullet$ & - & - & - & - & - & - & - & - & 48.6 & \#18 \\
\hline 9 & 0 & • & - & $\bullet$ & - & - & - & - & - & - & - & 77.0 & $\# 5$ \\
\hline 10 & 0 & • & • & - & - & - & - & - & $\bullet$ & - & - & 61.3 & \#11 \\
\hline 11 & 0 & $\bullet$ & - & $\bullet$ & - & - & - & - & - & - & - & 80.6 & \#4 \\
\hline 12 & 0 & • & • & - & - & - & - & - & - & - & - & 63.6 & $\# 10$ \\
\hline 13 & 0 & $\bullet$ & - & $\bullet$ & - & - & - & - & - & - & - & 84.3 & \#3 \\
\hline 14 & 0 & $\bullet$ & $\bullet$ & - & - & - & - & - & • & - & - & 74.0 & \#8 \\
\hline 15 & 0 & • & - & • & - & - & - & - & - & - & - & 85.6 & $\# 2$ \\
\hline 16 & 0 & • & • & - & - & - & - & - & - & - & - & 76.6 & \#6 \\
\hline 0 & 1 & - & - & - & - & - & - & $\bullet$ & - & - & - & 16.3 & \#34 \\
\hline Accur & & $88 \%$ & $83 \%$ & $73 \%$ & $56 \%$ & $80 \%$ & $48 \%$ & $92 \%$ & $82 \%$ & $86 \%$ & $92 \%$ & & \\
\hline Ranki & & $\# 3$ & \#5 & \#8 & \#9 & $\# 7$ & \#10 & \#1 & \#6 & $\# 4$ & $\# 1$ & & \\
\hline
\end{tabular}




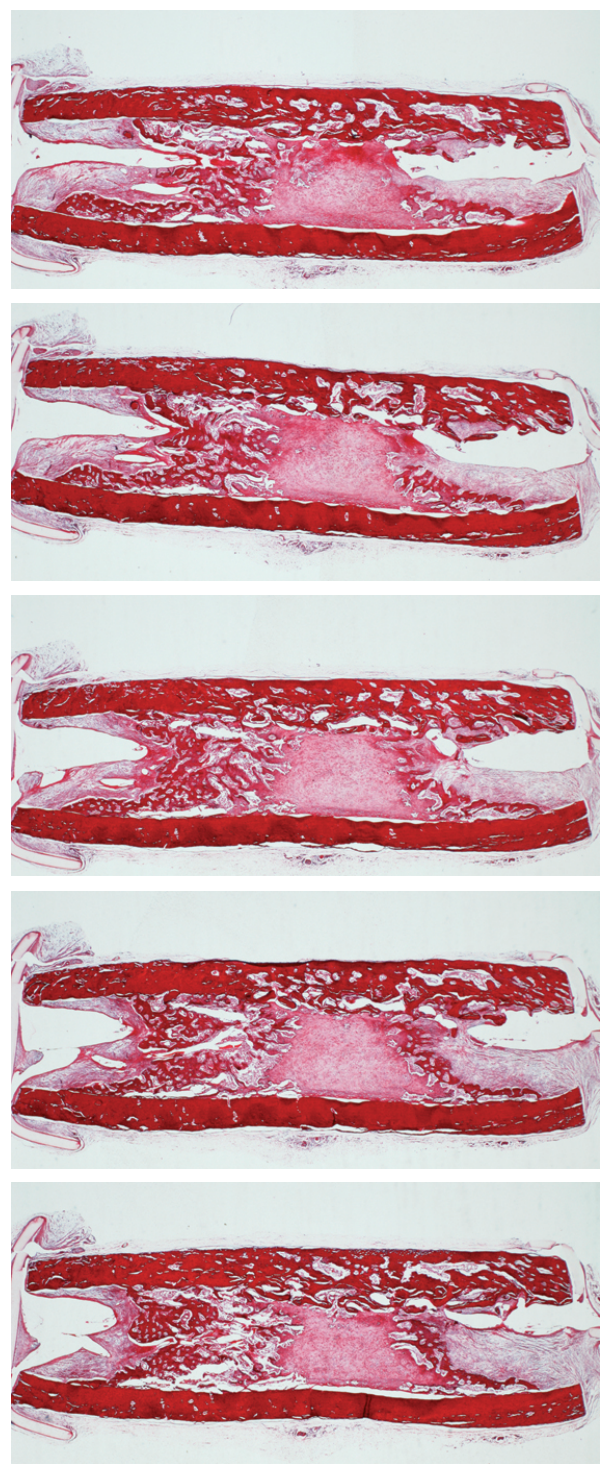

(a)
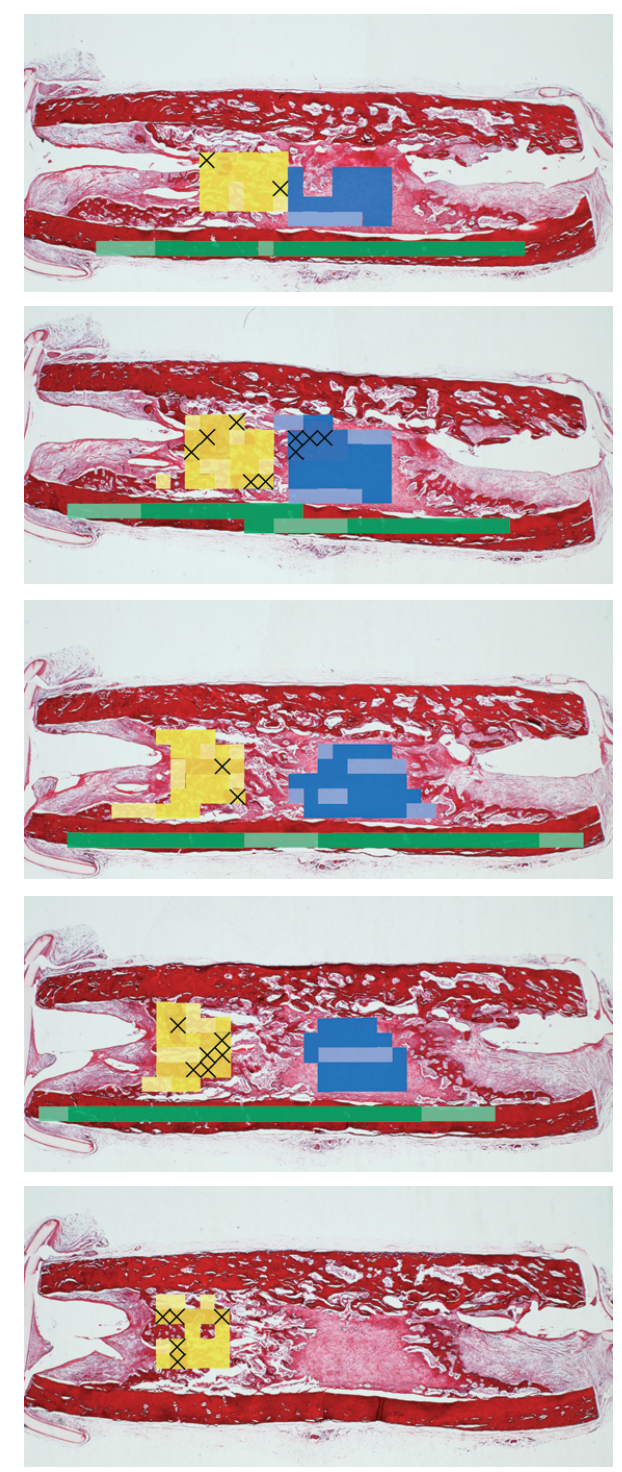

(b)
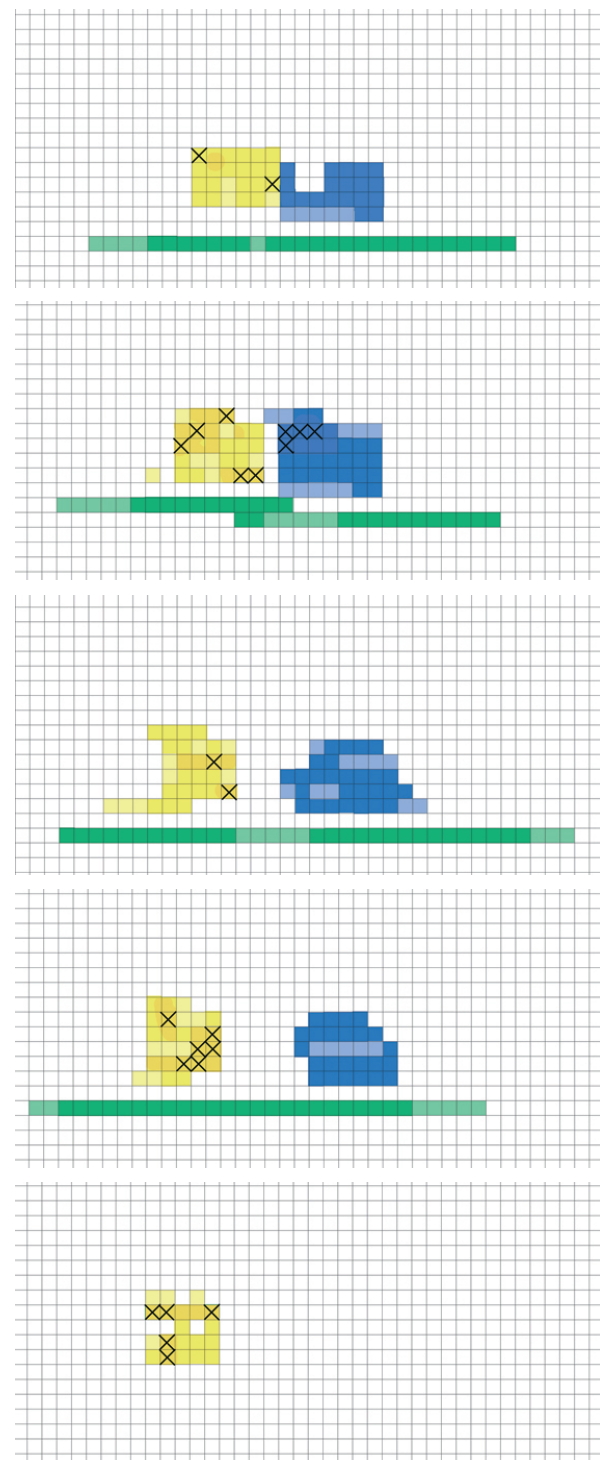

(c)

Figure 1: Five biomedical tissue images consecutively extracted from a 3D volume and stained with picrosirius were involved in our training and classification phases. The areas between strong red and spongy pink is where the regeneration of bone tissue occurs. (a) Original images, (b) old bone, cartilage and regenerated bone regions (green, purple and yellow areas located in the lower strip, central and left side, respectively), and (c) training samples and the tiles used during the classification process (darker colors). Overall, there are 130 samples for each type of tissue, 30 of them used for training our classifiers and the remaining 100 used for running the classification tests (those 24 tiles which are wrongly assigned by our most reliable classifier are marked with a cross, "X", to be depicted later in Figure 2). Image size is 2560x1920 pixels, tile size is 64x64 pixels (tiles containing white background are not shown).

into the 2D space to derive a vector composed of only $k$ components. This vector is compared against each of the samples, the vector norm is measured as distance, and the image is assigned to the class containing the sample with the minimum distance.

\section{CLASSIFICATION BASED ON $(\alpha-\beta)-K$-FEATURE SET}

The $(\alpha-\beta)-k$-feature set problem (3) combinatorial approach selects $k$ features where there are at least $\alpha$ features with different values for all pairs of tiles in distinct classes and at least $\beta$ features with the same value for all pairs of tiles within a class. In order to find a small set of features, we run the $(\alpha-\beta)$ - $k$-feature set problem with $\alpha=1$ and $\beta=0$ (a special problem known as $k$-feature set. The goal is then to find a solution of minimum cardinality, which is attained for this problem with $k=4$ as optimal solution. This number matches the best dimensionality reported by our previous PCA experiment and constitute a good value for a fair comparison. The two sets of four features which maximize the discriminant power for the classification process are $\left\{A_{0,0}, A_{13,6}, A_{44,4}, A_{45,30}\right\}$ and $\left\{A_{3,20}, A_{11,0}, A_{17,48}, A_{21,0}\right\}$. Using them as vectors of features we run again the classifier to test their accuracy on the distinction of three classes of bone tissue we are interested on, and compare the success with previous methods.

\section{EXPERIMENTAL RESULTS}

\subsection{Input data set and training samples}

Ir order to compare our different classification approaches, we selected five consecutive slides of 


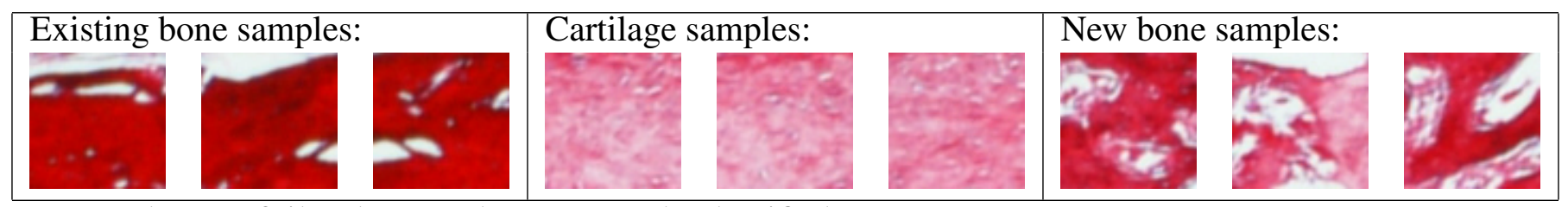

The set of tiles that are always wrongly classified:

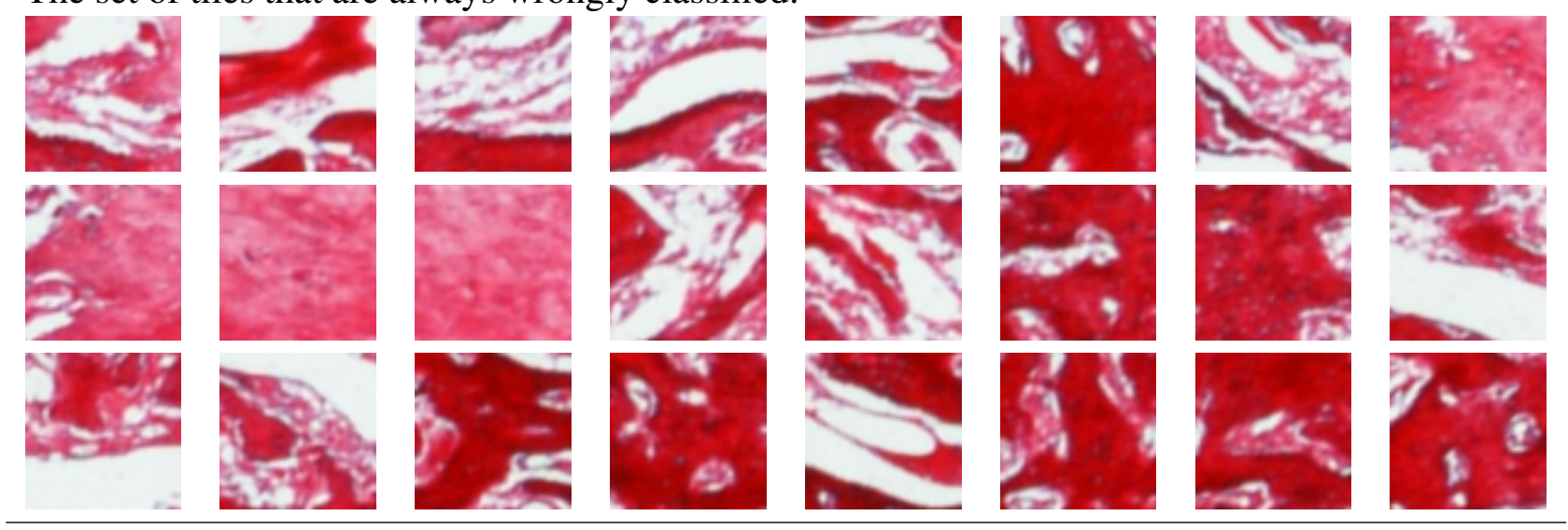

Figure 2: Upper side: Typical examples of each class used during the training phase of the classifiers. Lower side: The set of 24 tiles wrongly classified under the most reliable process, the one using our vector of features \#10 composed of a single Legendre moment, $A_{0,0}$. Those 24 tiles are also marked with a cross in Figure 1.c.

biomedical tissue from which 390 tiles, 130 of each class, were chosen by biomedical experts (see Figure 1): 30 of them for the training phase of each method, and the remaining 100 for the classification tests. These experts warned us about image areas where smooth transitions are taking place, and the distinction between existing and regenerated bone is rather fuzzy even for the experts. Therefore, we expect errors to happen around those hybrid zones.

\subsection{Classification accuracy}

Table 2 shows the percentage of success for classifying correctly the 300 input tiles, 100 for each type of tissue, together with a rank based on accuracy. In our more reliable classification trials (see vector of features \#7 and \#10), 20 tiles that were marked as new bone by our biomedical experts were wrongly classified, and 4 tiles or cartilage too. This set of challenging tiles are identified with crosses in Figure 1.c and shown with higher detail in Figure 2. Most of those tiles correspond to the class of new bone, presumably because they are on an early transition stage during the regeneration process departing from stem cells. Table 2 confirms on its last row that new bone is the more challenging class on average for a correct classification (just $54 \%$ ).

Among our three methods, the preliminary selection is the more reliable one, holding eight of the ten tests which best qualify (in particular, those having the ad hoc addition of $A_{0,0}$ ). On the other hand, PCA analysis produces five of the worst seven, and finally, the $(\alpha-\beta)-k$-feature set problem stays right in between in positions 8 and 9 out of 17 .

Regarding the importance of Legendre moments, we can extract four main conclusions:
1. Using a large vector of Legendre moments does not guarantee the success during the classification process, because they can be highly correlated. The most important issue, as our PCA analysis reveals, is to identify those moments which are more discriminant, and the best results are obtained for $k=4$, which also led us to consider exactly $k=4$ during our $(\alpha-\beta)$ - $k$-feature set method.

2. The set of Legendre moments composed of the last repetition for each order $\left(A_{0,0}, A_{1,1}, \ldots, A_{16,16}\right.$ (our vector of features \#4 in Table 2) retain less amount of information than those moments corresponding to the first repetition $\left(A_{0,0}, A_{1,0}, \ldots, A_{16,0}\right)$ (vector $\left.\# 1\right)$, and the ranking we provided on Table 1 for the discriminant power of each moment alone (see last column) confirms this observation.

3. The $A_{0,0}$ moment represents a key feature for a successful classification, as the vector of features \#10 is the leader of our rank, which is composed solely of this Legendre moment. Also, the worst 3 vectors of features in our ranking are precisely those which lack of this particular moment. Between the two vectors of features optimally chosen following the $\alpha-\beta$ - $k$-feature set method, however, the one excluding $A_{0,0}$ behaves slightly better.

4. At the cost of introducing other features, the only way to improve over the performance of a classifier that only uses $A_{0,0}$ is to select a different feature set which better differentiates the cases of existing bone and new bone, and new bone and cartilage. 
Table 2: Percentage of success for each of the classification methods. Test runs are placed on rows, output classes on columns. After a training phase tailored to each classification method, 100 samples of each class were fed to the classifier, for a total of 300 input tiles (64x64 pixels each) to be tagged either as cartilage, existing bone, or new (regenerated) bone.

\begin{tabular}{|c|c|c|c|c|c|}
\hline \multirow{2}{*}{$\begin{array}{l}\text { Selection of features } \\
\text { to run our classifier }\end{array}$} & \multicolumn{3}{|c|}{ Tissue correctly classified } & \multirow{2}{*}{$\begin{array}{l}\text { Average } \\
\text { success }\end{array}$} & \multirow{2}{*}{$\begin{array}{l}\text { Position in } \\
\text { our ranking }\end{array}$} \\
\hline & Cartilage & Bone & New bone & & \\
\hline \multicolumn{6}{|c|}{ Preliminary selection of moments based on previous experiences: } \\
\hline Vector of features \#1 & $90 \%$ & $90 \%$ & $84 \%$ & $88.00 \%$ & 3 \\
\hline Vector of features \#2 & $75 \%$ & $90 \%$ & $86 \%$ & $83.66 \%$ & 5 \\
\hline Vector of features \#3 & $97 \%$ & $93 \%$ & $30 \%$ & $73.33 \%$ & 10 \\
\hline Vector of features \#4 & $59 \%$ & $78 \%$ & $33 \%$ & $56.66 \%$ & 14 \\
\hline Vector of features \#5 & $85 \%$ & $92 \%$ & $64 \%$ & $80.33 \%$ & 7 \\
\hline Vector of features \#6 & $55 \%$ & $55 \%$ & $36 \%$ & $48.66 \%$ & 17 \\
\hline Vector of features \#7 & $96 \%$ & $100 \%$ & $80 \%$ & $92.00 \%$ & 1 \\
\hline Vector of features \#8 & $92 \%$ & $93 \%$ & $63 \%$ & $82.66 \%$ & 6 \\
\hline Vector of features \#9 & $96 \%$ & $100 \%$ & $64 \%$ & $86.66 \%$ & 4 \\
\hline Vector of features \#10 & $96 \%$ & $100 \%$ & $80 \%$ & $92.00 \%$ & 1 \\
\hline \multicolumn{6}{|c|}{ Using PCA analysis to reduce to $k$ dimensions the $17 \times 17$ features space: } \\
\hline$k=2$ dimensions & $54 \%$ & $74 \%$ & $45 \%$ & $57.66 \%$ & 13 \\
\hline$k=3$ dimensions & $48 \%$ & $74 \%$ & $46 \%$ & $56.00 \%$ & 15 \\
\hline$k=4$ dimensions & $51 \%$ & $81 \%$ & $50 \%$ & $60.66 \%$ & 11 \\
\hline$k=5$ dimensions & $48 \%$ & $81 \%$ & $46 \%$ & $58.33 \%$ & 12 \\
\hline$k=6$ dimensions & $49 \%$ & $78 \%$ & $39 \%$ & $55.33 \%$ & 16 \\
\hline \multicolumn{6}{|c|}{ Using $(\alpha-\beta)-k$-feature set problem to select the most discriminative set of four moments: } \\
\hline$A_{0,0}, A_{13,6}, A_{44,4}, A_{45,30}$ & $99 \%$ & $97 \%$ & $31 \%$ & $75.66 \%$ & 9 \\
\hline$A_{3,20}, A_{11,0}, A_{17,48}, A_{21,0}$ & $94 \%$ & $88 \%$ & $52 \%$ & $78.00 \%$ & 8 \\
\hline Degree of tissue recognized & $75.52 \%$ & $86.11 \%$ & $54.64 \%$ & & \\
\hline
\end{tabular}

\section{SUMMARY AND CONCLUSIONS}

In this work, we analyze the usefulness of Legendre moments as descriptors of biomedical images for quantifying the degree of bone tissue regeneration from stem cells. We have identified those moments which are more selective for an image segmentation into bone and cartilage regions, and applied three different techniques to reduce the computational complexity aiming to real-time applications.

Overall, low order moments and repetitions retain most of the relevant information as image descriptors at $64 \times 64$ pixels tile level, with the $A_{0,0}$ moment capturing most of the discriminant power. PCA analysis [3] did not show any significant progress for reducing the dimensionality of the problem, but pointed out an optimal dimensionality of four. These two observations were confirmed by our preselection of moments on a assorted set of 10 different vectors of features, where the ones having just the $A_{0,0}$ and a cardinality of four including $A_{0,0}$ showed the highest classification success. Finally, our combinatorial approach based on $(\alpha-\beta-k)$-feature set revealed low order moments and repetitions as the most effective features when the method was tailored to $\alpha=1, \beta=0$ and $k=4$ based on hints provided by counterpart methods.

\section{ACKNOWLEDGEMENTS}

This work was supported by the Junta de Andalucía under Project of Excellence P06-TIC-02109, the Min- istry of Education of Spain under Project TIN200601078, and mobility grants PR2011-0144 and PRX12-00053, and the Australian Research Council's Discovery Projects funding scheme (DP120102576).

\section{REFERENCES}

[1] Andrades JA, Santamaría J, Nimni M, Becerra J. 2001. Selection, amplification and induction of a bone marrow cell population to the chondroosteogenic lineage by rhOP-1. Intl. J. Dev. Biology 45:683-693.

2] Bin Y, Jia-Ziong P. 2002. Invariance Analysis of Improved Zernike Moments. J. Opt. A: Pure Appl. Opt 4(6):606.

3] Cotta C, Langston M, Moscato P. 2006. Combinatorial and Algorithmic Issues for Microarray Analysis. Handbook Approx. Algorithms and Metaheuristics. T.F. Gonzalez.

4] Khotanzad A, Hong Y. 1990. Invariant Image Recognition by Zernike Moments. IEEE Trans. on P.A.M.I. 12(5):489-497.

5] Martín-Requena MJ, Ujaldón M. 2011. Leveraging Graphics Hardware for an Automatic Classification of Bone Tissue. Book Computational Vision and Medical Image Processing - Recent Trends 19:209-228.

[6] Martínez A, Avinash C. 2001. PCA versus LDA. IEEE Trans. on P.A.M.I 23(2):228-233.

[7] Mukundan R, Ramakrishnan K. 1995. Fast Computation of Legendre and Zernike Moments. Pattern Recognition 28(9): 1433-1442.

[8] Teague M. 1980. Image Analysis via the General Theory of Moments. J. Opt. Society of America 70(8):920-930.

[9] Teh C, Chin R. 1998. On Image Analysis by the Methods of Moments. IEEE Trans. on P.A.M.I. 10:496-512. 